\title{
METRICAL AND TOPOLOGICAL PRESSURE OF FLOWS WITHOUT FIXED POINTS
}

\author{
Lianfa He, Fenghong Yang and Yinghui Gao
}

\begin{abstract}
We study the metrical and topological pressure for flows without fixed points on a compact metric space, and get the results as follows: (1) The metrical pressure with respect to an ergodic measure can be defined by $(t, \varepsilon)$-spanning sets. (2) The topological pressure is the supremum of metrical pressures with respect to all ergodic measures. (3) The properties that the topological pressure is zero, nonzero, finite or infinite respectively are invariant under weak equivalence.
\end{abstract}

\section{Introduction}

Let $(X, d)$ be a compact metric space with the metric $d$, and $\varphi$ : $R \times X \rightarrow X$ be a continuous flow(flow for short). Write $\varphi_{t}$ for the homeomorphism of $X$ defined by $\varphi_{t}(x)=\varphi(t, x)$. Define $\mathcal{B}$ to be the $\sigma$-algebra of Borel subsets of $X$. Let $\Phi$ represent $\varphi$ or $\varphi_{1}$. The set of all $\Phi$-invariant probability measures on $(X, \mathcal{B})$ is denoted by $M(\Phi)$. Put $E(\Phi)=\{\mu \in M(\Phi) \mid \mu$ is ergodic for $\Phi\} . C(X, R)$ denotes the Banach space of real-valued continuous functions on $X$ equipped with the supremum norm. For $m \in M(\varphi)$ and $f \in C(X, R)$ we define

$$
P_{m}(\varphi, f)=h_{m}\left(\varphi_{1}\right)+\int f d m,
$$

where $h_{m}\left(\varphi_{1}\right)$ is the metrical entropy of $\varphi_{1}$ (with respect to $m$ ).

$P_{m}(\varphi, f)$ is called to be the metrical pressure of $\varphi$ with respect to $f$ and $m$.

Received January 14, 2004.

2000 Mathematics Subject Classification: Primary 28D20; Secondary 28D10, $54 \mathrm{H} 20$.

Key words and phrases: flows, weak equivalence, metrical pressure, topological pressure. 
For $F, E \subset X$ we say $F(t, \varepsilon)$-spans $E$ (with respect to $\varphi$ ), if for each $x \in E$ there is a $y \in F$ such that

$$
d\left(\varphi_{s}(x), \varphi_{s}(y)\right) \leq \varepsilon, \forall s \in[0, t] .
$$

Topological pressure for a flow $\varphi$ with respect to $f$ is defined by Bowen in [1]. It is that

$$
P(\varphi, f)=\lim _{\varepsilon \rightarrow 0} \limsup _{t \rightarrow \infty} \frac{1}{t} \log P(\varphi, f, \varepsilon, t),
$$

where $P(\varphi, f, \varepsilon, t)=\inf \left\{\sum_{x \in F} \exp \int_{0}^{t} f \circ \varphi_{s}(x) d s \mid F(t, \varepsilon)-\right.$ spans $\left.X\right\}$. In particular, if $f=0$, then $P(\varphi, 0)$ is just the topological entropy for $\varphi$.

Given $\varphi$ and $\psi$ flows on $X$ and $Y$ respectively, we say $\varphi$ and $\psi$ are weakly equivalent if there is a homeomorphism $\lambda$ of $X$ onto $Y$ such that $\varphi$ and $\hat{\varphi}=\left\{\hat{\varphi}_{t}=\lambda^{-1} \circ \psi_{t} \circ \lambda: t \in R\right\}$ have the same orbits ([6]). We know from [9] that, for continuous maps on compact spaces, both topological entropy and topological pressure are invariant under conjugacy. However, this does not hold for mutually weakly equivalent flows in general. In [6], Ohno proved that the properties that the topological entropy is zero, positive, finite or infinite respectively are invariant under weak equivalence for flows without fixed points (Theorem 1 in [6]). $\mathrm{He}$ showed also that this is not case if the flows have fixed points ( Theorem 2 in $[6])$.

This paper is a further attempt to approach some problems of metrical and topological pressure for any flows without fixed points. In section 2, we shall give an equivalent definition of metrical pressure for an ergodic $\varphi$-invariant measure by $(t, \varepsilon)$-spanning sets (Theorem 1$)$. In section 3 , we shall prove that the topological pressure is the supremum of metrical pressures with respect to all ergodic $\varphi$-invariant measures (Theorem 2). In Section 4, we shall extend Theorem 1 in [6] to topological pressure (Theorem 3 ).

\section{Metrical pressure of flows with respect to ergodic mea- sures}

In [3], Katok gave an equivalent definition of metrical entropy of a homeomorphism with respect to an ergodic invariant measure by $(n, \varepsilon)$ spanning sets. The following adopts the Katok's ideas. 
For $m \in E(\varphi), f \in C(X, R)$ and $\delta \in(0,1)$. Put

and

$$
\begin{aligned}
Q_{m}(\varphi, f, \varepsilon, t, \delta)= & \inf \left\{\sum_{x \in F} \exp \int_{0}^{t} f \circ \varphi_{s}(x) d s \mid F \text { is a }(t, \varepsilon)\right. \\
& \text {-spanning set of a set of m-measure } \\
& \geq 1-\delta\}, \\
Q_{m}(\varphi, f, \varepsilon, \delta)= & \limsup _{t \rightarrow+\infty} \frac{1}{t} \log Q_{m}(\varphi, f, \varepsilon, t, \delta), \\
Q_{m}(\varphi, f)= & \lim _{\varepsilon \rightarrow 0} Q_{m}(\varphi, f, \varepsilon, \delta) .
\end{aligned}
$$

REMARK 1.

1. Theorem 1 we shall prove shows that the limit exists and is independent of $\delta$.

2. For any $c \in R$,

$$
Q_{m}(\varphi, f+c)=Q_{m}(\varphi, f)+c .
$$

THEOREM 1. Let $\varphi$ be a flow without fixed points on $X$. For $m \in$ $E(\varphi)$ and $f \in C(X, R)$, we have

$$
\begin{aligned}
Q_{m}(\varphi, f) & =h_{m}\left(\varphi_{1}\right)+\int f d m \\
& =P_{m}(\varphi, f) .
\end{aligned}
$$

Let $g: X \rightarrow X$ be a continuous map, and $\xi$ a finite measurable partition of $(X, \mathcal{B})$. Put

$$
\xi^{n}=\xi \vee g^{-1} \xi \vee \cdots \vee g^{-(n-1)} \xi, \forall n \geq 1 .
$$

For $x \in X$, let $A_{n}(x)$ denote the member of the partition $\xi^{n}$ to which $x$ belongs.

Lemma 1. ${ }^{4]}$ (Shannon-McMillan-Breiman Theorem) For $\mu \in M(g)$ and any finite measurable partition $\xi$ of $(X, \mathcal{B})$, there is a $\mu$-integrable function, denoted by $\mu_{\xi}$, such that

(1) $\lim _{n \rightarrow+\infty}\left(-\frac{1}{n} \log \mu\left(A_{n}(x)\right)\right)=\mu_{\xi}(x)$ a.e.,

(2) $\int \mu_{\xi}(x) d \mu=h_{\mu}(g, \xi)$,

where $h_{\mu}(g, \xi)$ is the entropy of $g$ with respect to $\xi$. 
Lemma 2. For a small constant $r>0$, let

$$
\begin{aligned}
\mathcal{A}_{n, r}^{-}(\xi) & =\left\{A_{n} \in \xi^{n} \mid \mu\left(A_{n}\right)<\exp \left(-n\left(h_{\mu}(g, \xi)-r\right)\right)\right\} \\
S_{n, r}^{-}(\xi) & =\bigcup_{A_{n} \in \mathcal{A}_{n, r}^{-}(\xi)} A_{n},
\end{aligned}
$$

then for some $\delta>0$, there is $N$ satisfying

$$
\mu\left(S_{n, r}^{-}(\xi)\right)>\delta, \text { for } n>N
$$

Proof. Write $X_{0}=S u p p(\mu)$, then $\mu\left(X_{0}\right)=1$. For $r>0$, we set $X_{r}$ $=\left\{y \in X_{0} \mid \mu_{\xi}(y)>h_{\mu}(g, \xi)-\frac{r}{2}\right\}$. Clearly, $\mu\left(X_{r}\right)>0$. Put $\mu\left(X_{r}\right)=2 \delta$. By Egoroff Theorem ([5]), there exists a measurable set $B \in \mathcal{B}$ such that $\mu(B)>1-\delta$, and the sequence $\left\{-\frac{1}{n} \log \mu\left(A_{n}(x)\right)\right\}$ converges uniformly to $\mu_{\xi}(x)$ on $B$. Therefore, for $\frac{r}{2}>0$ there is $N$ such that

$$
-\frac{1}{n} \log \mu\left(A_{n}(x)\right)>\mu_{\xi}(x)-\frac{r}{2}
$$

for every $x \in B$ and $n>N$. Notice that $\mu\left(B \cap X_{r}\right)>\delta$, so for any $y \in B \bigcap X_{r}$ and $n>N$, we have

$$
-\frac{1}{n} \log \mu\left(A_{n}(y)\right)>\mu_{\xi}(x)-\frac{r}{2}>h_{\mu}(g, \xi)-r,
$$

i.e.

$$
\mu\left(A_{n}(y)\right)<\exp \left(-n\left(h_{\mu}(g, \xi)-r\right)\right) .
$$

So $\left(A_{n}(y)\right) \in \mathcal{A}_{n, r}^{-}(\xi)$, and thus

$$
\mu\left(S_{n, r}^{-}(\xi)\right) \geq \mu\left(B \bigcap X_{r}\right)>\delta .
$$

Now the proof of the lemma is complete.

Similar to the proof of Lemma 2, we can get the following

LEMMA 3. For a small constant $r>0$, let

$$
\begin{aligned}
\mathcal{A}_{n, r}^{+}(\xi) & =\left\{A_{n} \in \xi^{n} \mid \mu\left(A_{n}\right)>\exp \left(-n\left(h_{\mu}(g, \xi)+r\right)\right)\right\} \\
S_{n, r}^{+}(\xi) & =\bigcup_{A_{n} \in \mathcal{A}_{n, r}^{+}(\xi)} A_{n},
\end{aligned}
$$

then there exist some $\delta>0$ and $N$ satisfying

$$
\mu\left(S_{n, r}^{+}(\xi)\right)>2 \delta, \text { for } n>N .
$$

Proof of Theorem 1, we give the proof in two steps.

Step 1. $Q_{m}(\varphi, f) \leq h_{m}\left(\varphi_{1}\right)+\int f d m$. 
For any $\sigma<0$, take $\varepsilon>0$ such that if $d(x, y)<\varepsilon$ then

$$
d\left(\varphi_{s}(x), \varphi_{s}(y)\right)<\sigma, \forall s \in[0,1],
$$

for all $x, y \in X$.

Since $m \in E(\varphi)$, it is easy to check $m \in M\left(\varphi_{1}\right)$ and is non-atomic. Take a finite partition $\xi$ of $X$ such that $\operatorname{diam}(\xi)<\frac{\varepsilon}{2}$. By Lemma 3, for $r>0$ there are $\eta>0$ and $N$ such that

$$
\mu\left(S_{n, r}^{+}(\xi)\right)>2 \eta, \text { for } n>N .
$$

It follows from $m \in E(\varphi)$ that

$$
\lim _{t \rightarrow+\infty} \frac{1}{t} \int_{0}^{t} f \circ \varphi_{s}(x) d s=\int f d m \text { a.e.. }
$$

Let $g_{n}(x)=\frac{1}{n} \int_{0}^{n} f \circ \varphi_{s}(x) d s$, then $g_{n} \rightarrow \int f d m$ a.e.. By Egoroff Theorem, for $\eta>0$ there is a measurable set $B$ such that $m(B)>1-\eta$, and $\left\{g_{n}\right\}$ converges uniformly to $\int f d m$ on $B$. Obviously $\frac{1}{t} \int_{0}^{t} f \circ \varphi_{s}(x) d s$ also converges uniformly to $\int f d m$ on $B$, and $m\left(E_{n}:=B \bigcap S_{n, r}^{+}(\xi)\right)>$ $\eta:=1-\delta, \forall n \geq N$.

Let

$$
Q_{m}(\varphi, f, \sigma, \delta)=\lim _{j \rightarrow+\infty} \frac{1}{t_{j}} \log Q_{m}\left(\varphi, f, \sigma, t_{j}, \delta\right) .
$$

We can put $\left\{t_{j}\right\}$ satisfying

$$
\left|\frac{1}{t} \int_{0}^{t} f \circ \varphi_{s}(x) d s-\int f d m\right|<\frac{1}{j}, \forall x \in B, t>t_{j} .
$$

Write $n_{j}=t_{j}+s_{j}, 0 \leq s_{j}<1$. Let $F_{n_{j}}$ be an $\left(n_{j}, \varepsilon\right)$-spanning set with the smallest cardinality of $E_{n_{j}}:=B \bigcap S_{n_{j}, r}^{+}(\xi)$ with respect to $\varphi_{1}$, then $F_{n_{j}}$ is a $\left(t_{j}, \sigma\right)$-spanning set of $E_{n_{j}}$ for $\varphi$. For any $A_{n_{j}} \in \xi^{n_{j}}$, we have $\operatorname{diam}\left(A_{n_{j}}\right)<\varepsilon$. So there is at most one element of $F_{n_{j}}$ in $A_{n_{j}}$, and therefore

$$
\operatorname{Card}\left(F_{n_{j}}\right) \leq \exp \left(n_{j}\left(h_{m}\left(\varphi_{1}, \xi\right)+r\right)\right) .
$$

By (3) and (4), we have

$$
Q_{m}\left(\varphi, f, \sigma, t_{j}, \delta\right) \leq \exp \left(n_{j}\left(h_{m}\left(\varphi_{1}, \xi\right)+r\right)+n_{j}\left(\int f d m+\frac{1}{j}\right)\right) .
$$

Hence

$$
\begin{aligned}
Q_{m}(\varphi, f, \sigma, \delta) & \leq h_{m}\left(\varphi_{1}, \xi\right)+r+\int f d m \\
& \leq h_{m}\left(\varphi_{1}\right)+r+\int f d m
\end{aligned}
$$


Let $r \rightarrow 0$ and then $\sigma \rightarrow 0$, we get

$$
Q_{m}(\varphi, f) \leq h_{m}\left(\varphi_{1}\right)+\int f d m
$$

Step 2. Put

$$
\begin{aligned}
q_{m}(\varphi, f, \varepsilon, \delta) & =\liminf _{t \rightarrow+\infty} \frac{1}{t} \log Q_{m}(\varphi, f, \varepsilon, t, \delta), \\
\text { and } \quad q_{m}(\varphi, f) & =\lim _{\varepsilon \rightarrow 0} q_{m}(\varphi, f, \varepsilon, \delta) .
\end{aligned}
$$

We shall prove $q_{m}(\varphi, f) \geq h_{m}\left(\varphi_{1}\right)+\int f d m$. that

Let $\xi=\left\{A_{1}, A_{2}, \ldots, A_{k}, A_{k+1}\right\}$ be a finite partition of $(X, \mathcal{B})$ such

1. $A_{1}, A_{2}, \ldots, A_{k}$ are pairwise disjoint and closed,

$$
\text { 2. } A_{k+1}=X \backslash \bigcup_{i=1}^{k} A_{i} \text {. }
$$

The inequality is a consequence of the following claim.

Claim. For any $r>0$ and any partition $\xi$ satisfying the properties 1.-2. above, it follows that

$$
r+q_{m}(\varphi, f) \geq h_{m}\left(\varphi_{1}, \xi\right)+\int f d m
$$

In order to prove the claim, we choose a positive integer $L$ so that $\frac{1}{L} \log 6<r$, and let

$$
\xi^{n}=\xi \vee \varphi_{L}^{-1} \xi \vee \cdots \vee \varphi_{L}^{-(n-1)} \xi
$$

Take a small constant $a>0$, by Lemma 2 , there are $\delta>0$ and $N$ such that

$$
m\left(S_{n, a}^{-}(\xi)\right)>4 \delta, \text { for } n>N
$$

Since

(7) $\liminf _{t \rightarrow+\infty} \frac{1}{t} \log Q_{m}(\varphi, f, \varepsilon, t, \delta)=\liminf _{n \rightarrow+\infty} \frac{1}{n L} \log Q_{m}(\varphi, f, \varepsilon, n L, \delta)$,

it is enough to prove the claim for $t=n L$.

For any $\sigma>0$, take $0<\varepsilon<\sigma$ such that if $d(x, y)<\varepsilon$ then

$$
\left|f \circ \varphi_{s}(x)-f \circ \varphi_{s}(y)\right|<\sigma,
$$

for any $x, y \in X$ and any $s \in[0,1]$.

Let $F_{n L}^{\prime}$ be an $(n L, \varepsilon)$-spanning set of $E$ of m-measure greater than or equal to $1-\delta$ with respect to $\varphi$, then it is an $(n, \varepsilon)$-spanning set of $E$ for $\varphi_{L}$. 
For $\delta>0$ and a set $B$ given by Egoroff Theorem, i.e. $m(B)>1-\delta$ and $\int_{0}^{t} f \circ \varphi_{s}(x) d s$ converges uniformly to $\int f d m(t \rightarrow+\infty)$ on $B$, let $E_{n L}:=B \cap E$, then

$$
m\left(E_{n L}\right)>1-2 \delta .
$$

Let $F_{n L} \subset F_{n L}^{\prime}$ be an $(n L, \varepsilon)$-spanning set with the smallest cardinality of $E_{n L}$ for $\varphi$. Let $N(1-\delta, \varphi, \varepsilon, n L)$ be the smallest cardinality of any $(n L, \varepsilon)$-spanning set of a set of m-measure greater than or equal to $1-\delta$ with respect to $\varphi$. Clearly

$$
N(1-\delta, \varphi, \varepsilon, n L) \geq N(1-2 \delta, \varphi, \varepsilon, n L) .
$$

It follows from the proof of Theorem 5 in [7] that

$$
\begin{aligned}
6^{n} \operatorname{Card}\left(F_{n L}^{\prime}\right) & \geq 6^{n} \operatorname{Card}\left(F_{n L}\right) \\
& \geq 6^{n} N(1-2 \delta, \varphi, \varepsilon, n L) \\
& \geq 2 \delta \exp \left(n\left(h_{m}\left(\varphi_{L}, \xi\right)-a\right)\right) .
\end{aligned}
$$

Choose $x_{n L} \in F_{n L}$ satisfying

$$
\int_{0}^{n L} f \circ \varphi_{s}\left(x_{n L}\right) d s=\min \left\{\int_{0}^{n L} f \circ \varphi_{s}(x) d s \mid x \in F_{n L}\right\},
$$

and notice that the cardinality of $F_{n L} \subset F_{n L}^{\prime}$ is the smallest, so there is $y_{n L} \in E_{n L}$ such that

$$
d\left(\varphi_{s}\left(x_{n L}\right), \varphi_{s}\left(y_{n L}\right)\right)<\varepsilon, \quad \forall s \in[0, n L] .
$$

By (8) and (11)-(13), we get

$$
\begin{aligned}
& 6^{n} \sum_{x \in F_{n L}^{\prime}} \exp \int_{0}^{n L} f \circ \varphi_{s}(x) d s \\
\geq & 2 \delta \exp \left(n\left(h_{m}\left(\varphi_{L}, \xi\right)-a\right)+\int_{0}^{n L} f \circ \varphi_{s}\left(y_{n L}\right) d s-n L \sigma\right) .
\end{aligned}
$$

Take $\left\{n_{j}\right\}$ such that

$$
\left|\frac{1}{n_{j}} \int_{0}^{n_{j} L} f \circ \varphi_{s}(x) d s-\int f d m\right|<\frac{1}{j}, \forall x \in B .
$$

and

$$
q_{m}(\varphi, f, \varepsilon, \delta)=\liminf _{j \rightarrow+\infty} \frac{1}{n_{j} L} \log Q_{m}\left(\varphi, f, \varepsilon, n_{j} L, \delta\right) .
$$


Therefore

$$
\begin{aligned}
6^{n} \sum_{x \in F_{n_{j} L}^{\prime}} \exp \int_{0}^{n_{j} L} f \circ \varphi_{s}(x) d s \geq & 2 \delta \exp \left(n _ { j } \left(h_{m}\left(\varphi_{L}, \xi\right)-a\right.\right. \\
& \left.\left.+L \int f d m-\frac{L}{j}-L \sigma\right)\right),
\end{aligned}
$$

and then

$$
\begin{aligned}
6^{n} Q_{m}\left(\varphi, f, \varepsilon, n_{j} L, \delta\right) \geq & 2 \delta \exp \left(n _ { j } \left(h_{m}\left(\varphi_{L}, \xi\right)-a+L \int f d m\right.\right. \\
& \left.\left.-\frac{L}{j}-L \sigma\right)\right) .
\end{aligned}
$$

Thus

$$
\frac{1}{L} \log 6+q_{m}(\varphi, f, \varepsilon, \delta) \geq \frac{1}{L}\left(h_{m}\left(\varphi_{L}, \xi\right)-a\right)+\int f d m-\sigma .
$$

Let $\sigma \rightarrow 0$ (so $\varepsilon \rightarrow 0$ ), we get

$$
q_{m}(\varphi, f)+r \geq \frac{1}{L}\left(h_{m}\left(\varphi_{L}, \xi\right)-a\right)+\int f d m .
$$

Notice that $\xi$ and $a$ are arbitrary and $h_{m}\left(\varphi_{L}\right)=L h_{m}\left(\varphi_{1}\right)$, therefore

$$
q_{m}(\varphi, f)+r \geq h_{m}\left(\varphi_{1}\right)+\int f d m .
$$

Now the proof of the theorem is complete.

Corollary 1. For any $m \in E(\varphi), \tau \in R \backslash\{0\}$ and $f \in C(X, R)$,

$$
P_{m}(\varphi, f)=\frac{1}{|\tau|} h_{m}\left(\varphi_{\tau}\right)+\int f d m
$$

\section{The variational principle of topological pressures}

For a given flow $\varphi$ on $X$ and $f \in C(X, R)$, we have known from [1] and the variational principle of topological pressure of continuous maps [9] that

$$
\begin{aligned}
P(\varphi, f) & =P\left(\varphi_{1}, f_{1}\right), \\
& =\sup \left\{h_{m}\left(\varphi_{1}\right)+\int f d m \mid m \in M\left(\varphi_{1}\right)\right\}, \\
& =\sup \left\{h_{m}\left(\varphi_{1}\right)+\int f d m \mid m \in E\left(\varphi_{1}\right)\right\} \\
& =\sup \left\{h_{m}\left(\varphi_{1}\right)+\int f d m \mid m \in M(\varphi)\right\},
\end{aligned}
$$


where $f_{1}(x)=\int_{0}^{1} f \circ \varphi_{s}(x) d s$, and $P\left(\varphi_{1}, f_{1}\right)$ is the topological pressure of $\varphi_{1}$ with respect to $f_{1}$.

Since there exist significant non-parallel gradients between $\varphi$ and $\varphi_{1}$, for instance, a $\varphi_{1}$-invariant measure is not $\varphi$-invariant in general, and an ergodic measure for $\varphi$ is not necessary ergodic for $\varphi_{1}$, it is reasonable to give the following.

THEOREM 2. Let $\varphi$ be a flow without fixed points on $X$. For any $f \in C(X, R)$, we have

$$
P(\varphi, f)=\sup \left\{h_{m}\left(\varphi_{1}\right)+\int f d m \mid m \in E(\varphi)\right\}
$$

Proof. It follows from (14) and $E(\varphi) \subset M(\varphi) \subset M\left(\varphi_{1}\right)$ that

$$
\sup \left\{h_{m}\left(\varphi_{1}\right)+\int f d m \mid m \in E(\varphi)\right\} \leq P(\varphi, f) .
$$

To prove the converse inequality, it is enough to prove

$$
\begin{aligned}
& \sup \left\{h_{m}\left(\varphi_{1}\right)+\int f d m \mid m \in E(\varphi)\right\} \\
\geq & \sup \left\{h_{m}\left(\varphi_{1}\right)+\int f d m \mid m \in E\left(\varphi_{1}\right)\right\} .
\end{aligned}
$$

For each $\mu \in E\left(\varphi_{1}\right)$ and $t \in R$, we define

$$
\begin{gathered}
\mu_{t}(B)=\mu\left(\varphi_{t}(B)\right), \text { for } B \in \mathcal{B} \\
m(B)=\int_{0}^{1} \mu_{t}(B) d t, \text { for } B \in \mathcal{B} .
\end{gathered}
$$

It is easily seen that $\mu_{t} \in E\left(\varphi_{1}\right)$ and $m \in E(\varphi)$.

Let $\operatorname{Supp}\left(\mu_{t}\right)(\operatorname{resp} . \operatorname{Supp}(m))$ denote the support set of $\mu_{t}$ (resp. $m$ ). By (15) and $\varphi_{t}: X \rightarrow X$ is a homeomorphism, one can easily show

$$
\varphi_{t}\left(\operatorname{Supp}\left(\mu_{t}\right)\right)=\operatorname{Supp}(\mu)
$$

and

$$
\operatorname{Supp}(m)=\overline{\bigcup_{t \in[0,1]} \operatorname{Supp}\left(\mu_{t}\right)}
$$

For $\delta \in(0,1)$ and $E_{m} \subset \operatorname{Supp}(m)$ whose m-measure is greater than or equal to $1-\delta$, suppose $F_{m}$ is a $(t, \varepsilon)$-spanning set of $E_{m}$ for $\varphi$. Put $t=n+s$, where $n$ is a positive integer and $s \in[0,1)$. It is easily seen from the equality (16) that there is $t_{0} \in[0,1]$ such that $\mu_{t_{0}}\left(E_{m}\right) \geq 1-\delta$. Put $E\left(t_{0}\right)=E_{m} \bigcap \operatorname{Supp}\left(\mu_{t_{0}}\right)$, then $\mu_{t_{0}}\left(E\left(t_{0}\right)\right) \geq 1-\delta$. We can see 
from the equality (17) that $\varphi_{t_{0}}\left(E\left(t_{0}\right)\right) \subset \operatorname{Supp}(\mu)$ and $\mu\left(\varphi_{t_{0}}\left(E\left(t_{0}\right)\right)\right)=$ $\mu_{t_{0}}\left(E\left(t_{0}\right)\right) \geq 1-\delta$. Write $E_{0}=\varphi_{t_{0}}\left(E\left(t_{0}\right)\right)$.

Since $F_{m}$ is a $(t, \varepsilon)$-spanning set of $E_{m}$ with respect to $\varphi, F_{0}:=$ $\varphi_{t_{0}}\left(F_{m}\right)$ is an $(n-1, \varepsilon)$-spanning set of $E_{0}$ for $\varphi_{1}$. By Remark 1, we can assume that $f \geq 0$. Therefore

$$
\begin{aligned}
\sum_{x \in F_{m}} \exp \int_{0}^{t} f \circ \varphi_{s}(x) d s & \geq \sum_{x \in F_{0}} \exp \int_{0}^{n-1} f \circ \varphi_{s}(x) d s \\
& =\sum_{x \in F_{m}} \exp \sum_{i=0}^{n-2} f_{1} \circ \varphi_{1}^{i}(x) \\
& \geq P_{\mu}^{*}\left(\varphi_{1}, f_{1}, \varepsilon, n-1\right),
\end{aligned}
$$

where $P_{\mu}^{*}\left(\varphi_{1}, f_{1}, \varepsilon, n-1\right)=\inf \left\{\sum_{x \in F} \exp \sum_{i=0}^{n-1} f_{1} \circ \varphi_{1}^{i}(x) \mid F\right.$ is a $(t, \varepsilon)$ spanning set of a set of $\mu$-measure $\geq 1-\delta$ with respect to $\varphi_{1}$ \} (see [2]).

Since $E_{m}$ is arbitrary, we have

$$
Q_{m}(\varphi, f, \varepsilon, t, \delta) \geq P_{\mu}^{*}\left(\varphi_{1}, f_{1}, \varepsilon, n-1\right) .
$$

It follows from Theorem 2.1 in [2] and Theorem 1 that

$$
h_{m}\left(\varphi_{1}\right)+\int f d m \geq h_{\mu}\left(\varphi_{1}\right)+\int f d \mu .
$$

This proves

$$
\sup \left\{P_{m}\left(\varphi_{1}, f\right) \mid m \in E(\varphi)\right\} \geq \sup \left\{P_{m}\left(\varphi_{1}, f\right) \mid m \in E\left(\varphi_{1}\right)\right\} .
$$

Now the proof of the theorem is complete.

\section{Topological pressures of mutually weakly equivalent flows}

THEOREM 3. Let $\varphi$ and $\psi$ be flows on compact metric spaces $X$ and $Y$ respectively. If $\varphi$ and $\psi$ are weakly equivalent and they have no fixed points, then we have

$$
P(\varphi, f)=C_{\varphi \psi} P\left(\psi, f \circ \lambda^{-1}\right), \text { for } f \in C(X, R),
$$

where $C_{\varphi \psi}$ is a constant, and $\lambda$ is the homeomorphism in the definition of weak equivalence.

Proof. Let $\hat{\varphi}=\left\{\hat{\varphi}_{t}=\lambda^{-1} \circ \psi_{t} \circ \lambda: t \in R\right\}$. It is trivial from the definition of weak equivalence that

$$
P(\hat{\varphi}, f)=P\left(\psi, f \circ \lambda^{-1}\right), \text { for } f \in C(X, R) .
$$


It is enough to prove that there exists a constant $C_{\varphi \psi}$ such that

$$
P(\varphi, f)=C_{\varphi \psi} P(\hat{\varphi}, f), \text { for } f \in C(X, R) .
$$

By Theorem 2, we have

$$
P(\varphi, f)=\sup \left\{h_{m}\left(\varphi_{1}\right)+\int f d m \mid m \in E(\varphi)\right\}
$$

and

$$
P(\hat{\varphi}, f)=\sup \left\{h_{\hat{m}}\left(\hat{\varphi}_{1}\right)+\int f d \hat{m} \mid \hat{m} \in E(\hat{\varphi})\right\} .
$$

In addition, we get from Definition 2.2 in [8] and Proposition 1 in [6] that there exist continuous functions $\theta: X \times R \rightarrow R$ and $\hat{\theta}: Y \times R \rightarrow R$ such that for each $m \in E(\varphi)$,

$$
\int f d \hat{m}=\frac{1}{\int \theta(1, x) d m} \int\left(\int_{0}^{\theta(1, x)} f \circ \hat{\varphi}_{s}(x) d s\right) d m, \text { for } f \in C(X, R),
$$

defines an $\hat{m} \in E(\hat{\varphi})$, the map $\Lambda: E(\varphi) \rightarrow E(\hat{\varphi}), m \mapsto \hat{m}$ is bijective, and the inverse map $\Lambda^{-1}: E(\hat{\varphi}) \rightarrow E(\varphi), \hat{m} \mapsto m$ is defined by

$$
\int f d m=\frac{1}{\int \hat{\theta}(1, x) d \hat{m}} \int\left(\int_{0}^{\hat{\theta}(1, x)} f \circ \varphi_{s}(x) d s\right) d m, \text { for } f \in C(X, R) .
$$

It follows from [6] that

$$
h_{\hat{m}}\left(\hat{\varphi}_{1}\right)=\frac{1}{\int \theta(1, x) d m} h_{m}\left(\varphi_{1}\right) .
$$

Therefore

$$
\frac{1}{A} h_{m}\left(\varphi_{1}\right) \leq h_{\hat{m}}\left(\hat{\varphi}_{1}\right) \leq \frac{1}{a} h_{m}\left(\varphi_{1}\right),
$$

where $A=\max \{\theta(1, x) \mid x \in X\}, a=\min \{\theta(1, x) \mid x \in X\}$. It is easily checked by (18) and (19) that

$$
C_{1} \int f d m \leq \int f d \hat{m} \leq C_{2} \int f d m
$$

where $C_{1}=\frac{b}{B}, B=\max \{\hat{\theta}(1, y) \mid y \in Y\}, b=\min \{\hat{\theta}(1, y) \mid y \in Y\}$, and $C_{2}=\frac{a}{A}$. 
Combining (20) with (21), we have that there exist constants $D_{1}$ and $D_{2}$ satisfying

$$
\begin{aligned}
D_{1}\left(h_{m}\left(\varphi_{1}\right)+\int f d m\right) & \leq h_{\hat{m}}\left(\hat{\varphi}_{1}\right)+\int f d \hat{m} \\
& \leq D_{2}\left(h_{m}\left(\varphi_{1}\right)+\int f d m\right) .
\end{aligned}
$$

It follows that

$$
D_{1} P(\varphi, f) \leq P(\hat{\varphi}, f) \leq D_{2} P(\varphi, f),
$$

and so there is a constant $C_{\varphi \psi}$ such that

$$
P(\varphi, f)=C_{\varphi \psi} P(\hat{\varphi}, f), \quad \text { for } f \in C(X, R) .
$$

Now the proof of the theorem is complete.

CorollaRY 2. ${ }^{[6]}$ If $f \equiv 0$ then $h(\varphi)=C_{\varphi \psi} h(\psi)$.

\section{References}

[1] R. Bowen and D. Ruelle, The ergodic theory of Axiom A flows, Invent. Math. 29 (1975), 181-202.

[2] Lianfa He, Jinfeng Lv and Lina Zhou, Definition of measure-theoretic pressure using spanning sets, to appear in Acta Math. Sin.

[3] A. Katok, Lyapunov exponents, entropy and periodic points for diffeomorphisms, Publ. IHES. 51 (1980), 137-173.

[4] R. Manè, Ergodic theory and differential dynamics, Springer, New-York. Berlin, 1987.

[5] M. E. Munroe, Introduction to measure and integration, Addison-wesley Publishing Company Inc. Cambridge, 42, MA. 1953.

[6] T. Ohno, A weak equivalence and topological entropy, Plub. Res. Inst. Math. Sci., Kyoto Univ. 16 (1980), 289-298.

[7] Wenxiang Sun and Edson Varges, Entropy of flows, revisited, Bol. Soc. Brasil. Mat. 30 (1999), 315-333.

[8] R. Thomas, Stability properties of one parameter flows, Proc. London Math. Soc. 45 (1982), 479-505.

[9] P. Walters, An introduction to ergodic theory, Springer-Verlag, New-York, Heidelberg, Berlin, 1982.

Lianfa He

Department of Mathematics

Hebei normal University

Shijiazhuang, 050016, P. R. China 


\section{Fenghong Yang}

Department of Mathematical Sciences

Tsinghua University

Beijing, 100084, P. R. China

E-mail: yfg01@mails.tsinghua.edu.cn

Yinghui Gao

Academy of Mathematics and System Sciences

Chinese Academy of Sciences

Beijing, 100080, P. R. China 
